\title{
Editorial
}

\section{Fault Diagnosis and Application to Modern Systems}

\author{
Xiao He, ${ }^{1}$ Zidong Wang, ${ }^{2}$ Gang Li, ${ }^{3}$ Zhijie Zhou, ${ }^{4}$ and Youqing Wang ${ }^{5}$ \\ ${ }^{1}$ Department of Automation, TNList, Tsinghua University, Beijing 100084, China \\ ${ }^{2}$ Department of Computer Science, Brunel University London, London UB8 3PH, UK \\ ${ }^{3}$ School of Instrument Science and Opto-Electronics Engineering, Beihang University, Beijing 100191, China \\ ${ }^{4}$ Xian High-Tech Institute, Xian 710025, China \\ ${ }^{5}$ College of Electrical Engineering and Automation, Shandong University of Science and Technology, Qingdao 266590, China \\ Correspondence should be addressed to Xiao He; hexiao@tsinghua.edu.cn
}

Received 27 April 2017; Accepted 27 April 2017; Published 24 May 2017

Copyright (c) 2017 Xiao He et al. This is an open access article distributed under the Creative Commons Attribution License, which permits unrestricted use, distribution, and reproduction in any medium, provided the original work is properly cited.

With the increasing demand for higher performance and higher safety and reliability standards, fault diagnosis (FD) for modern control systems has become an active field of research over the past decades. FD is a theory and technology that can utilize the analytical redundancy relationship of the control system and provide the whole system with accurate information of the fault that occurred by using the input and output of the system. Both theoretical challenge and practical application demands of many kinds of real-time control systems motivate the investigation of FD. A typical FD process consists of a fault detection unit, a fault isolation strategy, and a fault estimation technique. The task of fault detection problem is to construct a residual signal which is then compared with a predefined threshold. When the residual exceeds the threshold, an alarm is generated. The scope of the fault isolation problem is to locate the true fault from all possible faults. The objective of the fault estimation problem is to determine the fault amplitude as well as the emergence time of the fault. The precise understanding of the fault that occurred by using FD technology is a prerequisite for the next fault accommodation process and thus is very important for system safety.

The existing FD techniques can be generally divided into two main categories: the model-based method and the data-driven approach. In the case where a mathematical model can be obtained for an objective system, a modelbased FD method can provide exact decoupling or maximum attenuation to the factors except the target fault. On the other hand, when it is hard to obtain a mathematical model but enough historical data of a system can be obtained, the datadriven approach is more applicable and may get a better FD result. Recently, analytical-based FD techniques for modern systems have received more and more attention and there have been an increasing number of results reported in the literature for the topics of FD. This special issue aims to bring together some typical recent developments in fault diagnosis technology and its application to modern systems. We have solicited submissions to this special issue from scholars from all over the world and have got 26 submissions. After rigorous peer-review processes, 9 papers have been selected to be published in this special issue.

Some works in this special issue focus on the modelbased FD and Fault-Tolerant Control (FTC) techniques. In the paper entitled "Detection of Intermittent Fault for Discrete-Time Systems with Output Dead-Zone: A Variant Tobit Kalman Filtering Approach" by J. Huang and X. He, a fault detection method for discrete-time systems with output dead-zone is proposed. Most of the present research on fault diagnosis focuses on input dead-zone instead of output dead-zone, where the dead-zone is usually treated as an unknown input. The Tobit Kalman filtering approach is employed in the design of the residual generator. Compared with the traditional Kalman filter, the Tobit Kalman filter avoids the estimation bias that is brought about by ignoring the correlation between the state and the measurement noise in the dead-zone. Due to the obvious discontinuity occurring in thresholds of the dead-zone, the gradient does not exist, which makes the Extended Kalman filter hard to apply. Also, when the output dead-zone is located between the sigma points, there will be a bias in the measurement noise covariance that causes a bias in the estimation using Unscented Kalman filter. By contrast, the above conditions 
can be averted by utilizing the Tobit Kalman filter that provides the optimal estimation with minimum variance when the output dead-zone appears. Even though the particle filter can give an accurate estimation in the output deadzone, the Tobit Kalman filter has similar computational expenses to the traditional Kalman filter to avoid the collapse that may well happen in applying a particle filter in the high dimensional systems. Therefore, the fault detection method proposed in this paper has higher practicability in modern control systems, especially the ones with limited computational power, like embedded systems. Meanwhile, the Tobit Kalman filter performs well in tracking so that the residual generator in this paper is capable of quickly and accurately detecting the appearance and disappearance of faults.

In the paper entitled "Fault-Tolerant Control of a Nonlinear System Actuator Fault Based on Sliding Mode Control" by J. He et al., a fault-tolerant control scheme is presented for a class of nonlinear systems with actuator faults and unknown input disturbances. The sliding mode control law is designed based on the reaching law method. Then, in view of the unpredictable state variables and unknown information in the control law, the original system is transformed into two subsystems through a coordinate transformation. One subsystem only has actuator faults, and the other subsystem has both actuator faults and disturbances. A sliding mode observer is designed for the two subsystems, respectively, and the equivalence principle of the sliding mode variable structure is used to realize the accurate reconstruction of the actuator faults and disturbances. The observation value and the reconstruction value are used to carry out an online adjustment to the designed sliding mode control law, and fault-tolerant control of the system is realized. Simulation results show the effectiveness of the proposed method.

Some works in this special issue pay attention to datadriven FD. Traditional data-driven fault diagnosis techniques are not applicable in the case with big data generated from the modern complicated industry process. In the paper entitled "A Novel Multimode Fault Classification Method Based on Deep Learning" by F. Zhou et al., a fault diagnosis method based on a hierarchical deep neural network is proposed. The hierarchical deep neural network consists of three hierarchies (each one is a traditional unsupervised multilayer neural network), which are utilized to calculate the running mode, to identify the fault component, and to classify the fault level of the research object, respectively. This method guarantees the accuracy of fault diagnosis. The proposed neural network has been applied to real sampled data generated by the Case Western Reserve University Bearing Data Center and the results demonstrate its efficiency compared with those of the hierarchical back propagation neural network, the support vector machine, and the deep neural network. Furthermore, the proposed algorithm is robust for its ability to handle both the big data and the sample in small scale, which overmatches the deep neural network for data in big scale.

Due to the complexity of the industrial process, the fault data usually has time-correlated characteristic and its distribution law is hard to obtain. This renders the traditional back propagation single-hidden-layer neural network unable to guarantee the accuracy of the fault classification. To solve this problem, in the paper entitled "Research on Fault Diagnosis Method Based on Rule Base Neural Network" by Z. Ni et al., a neural network fault diagnosis method based on rule base is proposed. It utilizes the known factors causing the faults to regularize the experts' experience by fuzzy logic and applies its rule base to the multi-hidden-layer neural network. This treatment can make full use of a priori knowledge, so it can guarantee the accuracy of neural network based fault diagnosis techniques. The proposed algorithm is robust to the size of the test data and is also applicable in the case where only data in small scale can be obtained. It can extract the effective samples without the assumption of the large-scale samples with identical distribution. The proposed algorithm not only is able to isolate a single fault but also can hold composite faults. By applying fuzzy logic, uncertain information is processed effectually. Thus, compared with other neural network based fault diagnosis approaches, the proposed method in this paper can obtain a more precise fault rule base and a higher classification accuracy.

As a widely used computing service method for largescale resource scheduling, cloud computing has gained momentum recently. In order to assess the reliability of cloud computing accurately and effectively, in the paper entitled "Reliability Assessment of Cloud Computing Platform Based on Semiquantitative Information and Evidential Reasoning" by H. Wei and P.-L. Qiao, a novel assessment approach for the cloud computing platform is presented. A new model of the reliability assessment based on ER rule is proposed, which can combine both of the qualitative knowledge and the quantitative data. In particular, the quantitative data include the Mean Time to Failures (MTTF), frequency of network attack, fault-tolerant rate of cloud computing platform, and Mean Time to Repair (MTTR) of service, and qualitative knowledge is associated with the subjective quality of a situation or phenomenon, such as scalability of cloud system, controllability of access terminal, completeness of service, and stability of service. A four-level reliability attributes structure of cloud computing platform is established, including "excellent," "good," "common," and "bad." In addition, the reliability of the cloud computing platform is categorized in four distinct aspects, that is, network reliability, hardware reliability, software reliability, and service reliability. A case study for assessing the reliability of an actual cloud computing platform is given, and its good performance on assessing the reliability of a real cloud computing platform sheds light on the effectiveness and rationality of the proposed method.

Considering that the partial least squares (PLS) model is based on the assumption of a single operating mode, in the paper entitled "An Efficient Quality-Related Fault Diagnosis Method for Real-Time Multimode Industrial Process" by K. Peng et al., a novel multimode PLS model is developed using the well-known Gaussian mixture model and PLS quality-related process monitoring problem is investigated for the multimode process based on finite Gaussian mixture models and PLS method. The advantage of original PLS is subsequently followed to achieve the quality-related monitoring goals. A Gaussian mixture model is implemented for training data to conduct the multimode division and estimate 
the model parameter set. Then, posterior probabilities of each monitored sample belonging to all Gaussian modes are calculated through the Bayesian inference strategy. Based on the posterior probabilities, a comprehensive statistics index is defined and a combined index was developed for the fault detection purpose. Finally, a new index named gradient contribution rate is proposed to measure the contribution to the combined index and find out the variable that should be in charge of fault in quality. Performances of the whole proposed scheme are verified in a real industrial hot strip mill process compared with some existing methods.

In practical industry applications, the collected process variables always have different units. In order to obtain a consistent result, traditional Principal Component Analysis (PCA) methods need to normalize the collected data to zero mean and unit variance first, which actually neglects some useful information between different variables. To shorten such a gap, in the paper entitled "Fault Diagnosis Method Based on Information Entropy and Relative Principal Component Analysis" by X. Xu and C. Wen, relativetransformation PCA is implemented based on information entropy instead of prior information to form a new fault diagnosis method called information-entropy relativetransformation PCA. The algorithm calculates the information entropy for each characteristic variable in the original dataset based on the information gain algorithm. According to the information entropy, it allocates the weight for each standardized characteristic variable. Relative-transformation PCA is utilized to obtain the features for fault diagnosis. Simulation experiments based on Tennessee Eastman process and Wine datasets demonstrate the feasibility and effectiveness of the new method.

Some works focus on the application of FD techniques for some specific real-time systems. For example, in the paper entitled "Neural Network Based Fault Detection and Diagnosis System for Three-Phase Inverter in Variable Speed Drive with Induction Motor" by F. Asghar et al., the fault detection and isolation problem is considered for three-phase inverters based on features extraction and neural networks. Six different single switching device open faults and six different double switching device open faults are considered. In order to present decorrelation between different kinds of faults, Clarke transformation is used to extract the main features of output currents of a three-phase inverter. Then, the extracted features are used to diagnose faults by using artificial neural networks. The proposed method is able to detect and isolate both single faults and multiple faults. Compared with the previous approaches, more accurate fault diagnosis results can be obtained by using the proposed method due to the application of feature extraction. Finally, simulations and experimental results demonstrate the effectiveness of the fault diagnosis scheme. The main novelty of the paper lies in the application of the Clarke transformation and the conduction of the experiment.

In the paper entitled "Intelligent Vehicle Embedded Sensors Fault Detection and Isolation Using Analytical Redundancy and Nonlinear Transformations" by N. Pous, a fault detection and isolation scheme is proposed with intelligent vehicle embedded sensors based on analytical redundancy and nonlinear transformations. Four kinds of faults, that is, additive and stuck odometric distance faults and multiple and stuck odometric velocity faults, are considered. The analytical redundancy models of the foregoing sensors are developed. A measure estimation method is proposed by using a measurement, which is sensitive to fault. By using the estimated measurements and the real-time measurements, the residual is designed based on a nonsymmetrical Gaussian transformation. Then, fault detection and isolation can be achieved by comparing the residuals and a predefined threshold. Experimental results illustrate the effectiveness of the fault diagnosis scheme.

Xiao $\mathrm{He}$

Zidong Wang Gang Li

Zhijie Zhou

Youqing Wang 


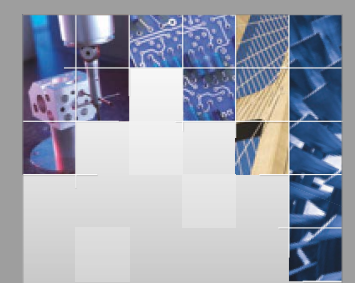

\section{Enfincering}
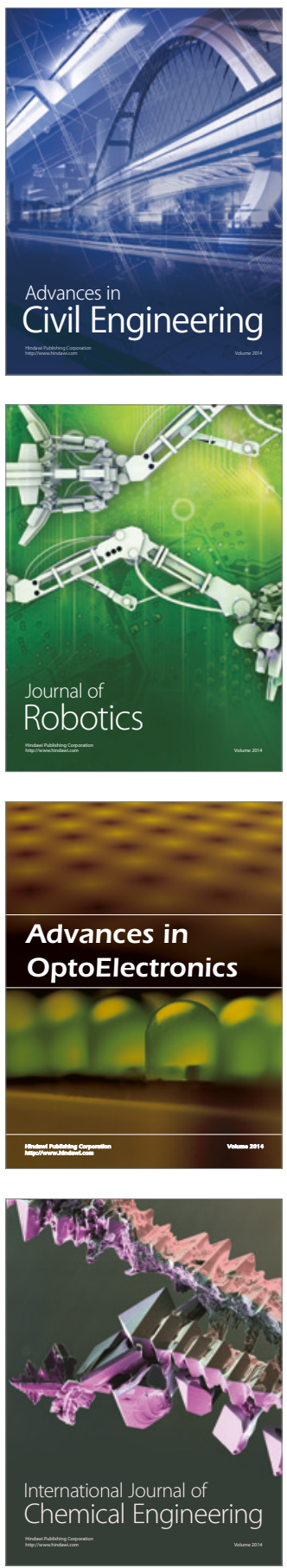

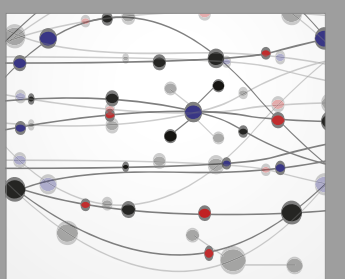

The Scientific World Journal

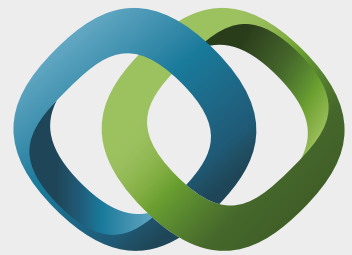

\section{Hindawi}

Submit your manuscripts at

https://www.hindawi.com
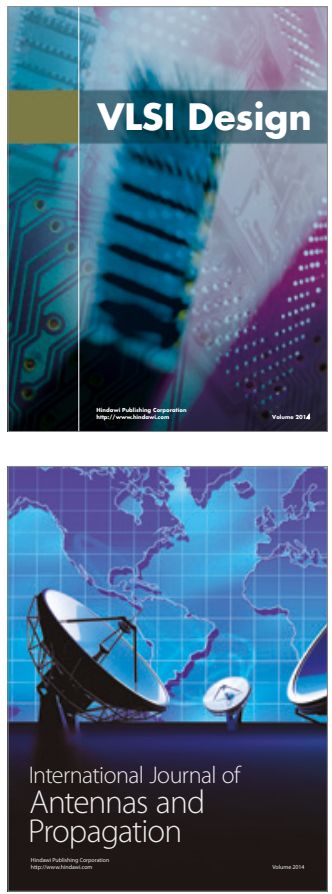

\section{Rotating}

Machinery
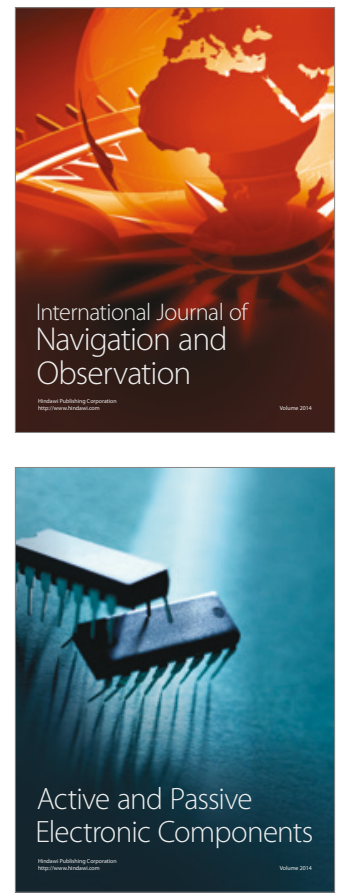
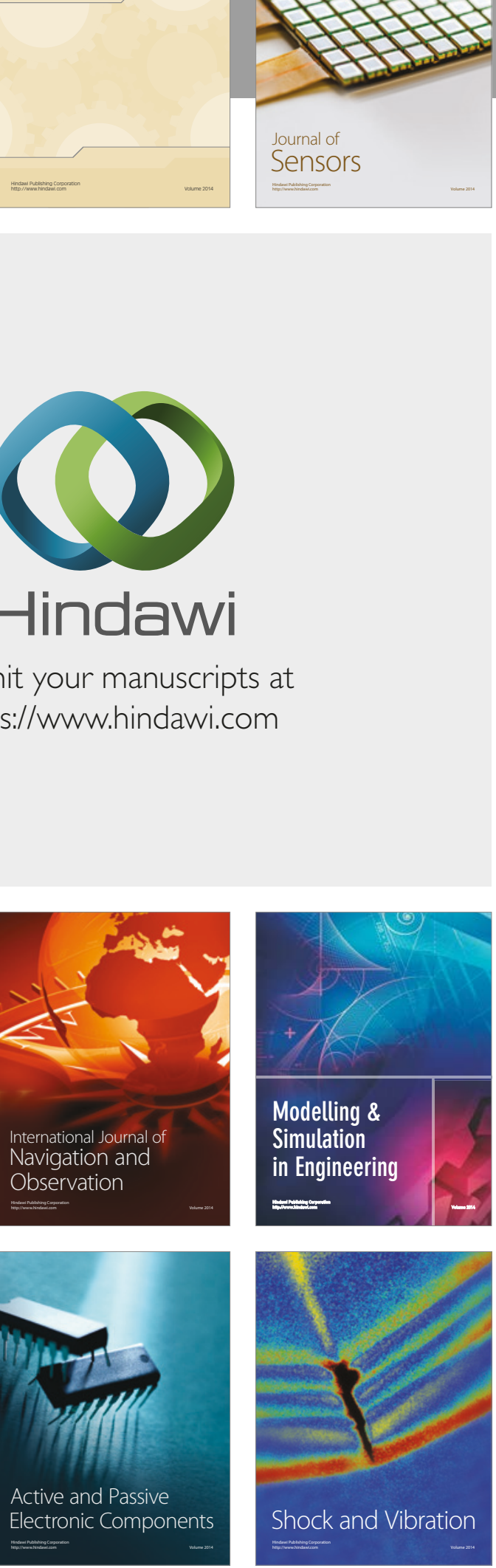
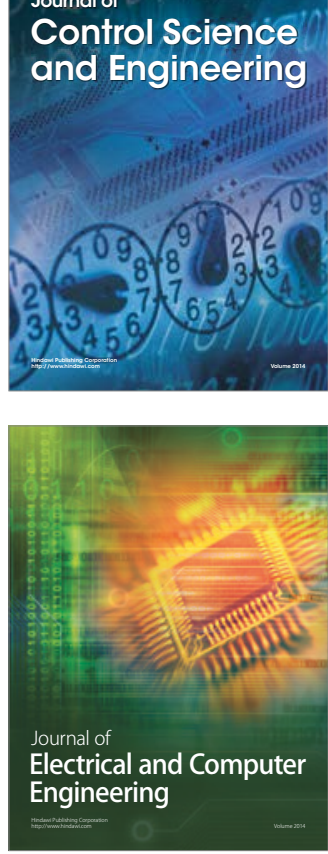

Distributed

Journal of

Control Science

and Engineering
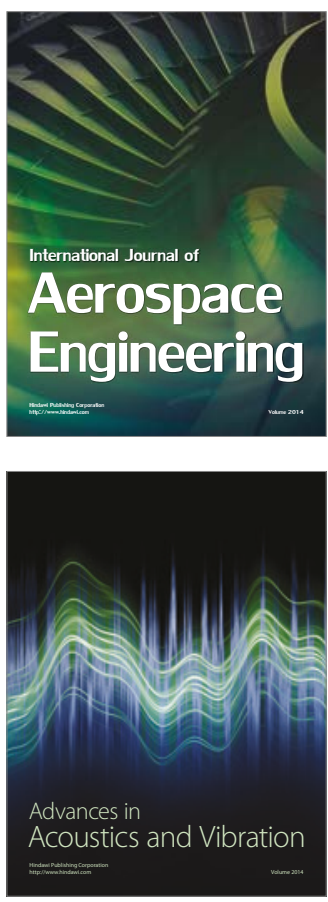

Sensor Networks 